\title{
Efficacy of an outer membrane protein of Pasteurella haemolytica A2, A7 or A9- enriched vaccine against intratracheal challenge exposure in sheep
}

\begin{abstract}
The outer membrane proteins (OMP) were extracted from the P. haemolytica A2, A7 and A9 to determine their potential as immunogens and their capability for cross-protection. Sixty lambs of approximately 9 months old were divided into four main groups. Animals in Group 1 were vaccinated with $2 \mathrm{ml}$ vaccine containing $100 \mathrm{mg} / \mathrm{ml}$ of the outer membrane proteins of P. haemolytica A2. Animals in Group 2 were similarly vaccinated with the OMPs of P. haemolytica A7 while Group 3 with OMPs of P. haemolytica A9. Animals in Group 4 were unvaccinated control. During the course of the study, serum was collected to evaluate the antibody levels toward each OMP. There appeared to be good immune responses. However, high antibody levels did not necessarily result in good protection of the animals, particularly against cross-infection with $\mathrm{P}$. haemolytica $\mathrm{A} 9$ in animals vaccinated with the OMPs of $\mathrm{P}$. haemolytica A2. It seemed that the antibody responses were more speci®c toward the homologous challenge but generally did not cross-protect against heterologous serotype challenge. However, the OMPs of P. haemolytica A7 produced good in vivo cross-protection and excellent correlations when good antibody responses against all serotypes led to successful reductions of the extent of lung lesions following homologous and heterologous challenge exposures. Thus, the OMPs of P. haemolytica A7 was effective in protecting animals against homologous and heterologous infection by live P. haemolytica A2, A7 and A9.
\end{abstract}

Keyword: Outer membrane proteins, Pasteurella haemolytica, Vaccine, Sheep 